\title{
Improving Difference Operators by Local Feature Detection
}

\author{
Kristof Teelen and Peter Veelaert \\ University College Ghent - Ghent University Association, \\ Schoonmeersstraat 52, B9000 Ghent, Belgium \\ \{Kristof.Teelen, Peter.Veelaert\}@hogent.be
}

\begin{abstract}
Differential operators are required to compute several characteristics for continuous surfaces, as e.g. tangents, curvature, flatness, shape descriptors. We propose to replace differential operators by the combined action of sets of feature detectors and locally adapted difference operators. A set of simple local feature detectors is used to find the fitting function which locally yields the best approximation for the digitized image surface. For each class of fitting functions, we determine which difference operator locally yields the best result in comparison to the differential operator. Both the set of feature detectors and the difference operator for a function class have a rigid mathematical structure, which can be described by Groebner bases. In this paper we describe how to obtain discrete approximates for the Laplacian differential operator and how these difference operators improve the performance of the Laplacian of Gaussian edge detector.
\end{abstract}

\section{Introduction}

A delicate and often reoccurring problem in digital image processing is the application of operators from differential geometry to digital representations of curves and surfaces. For continuous surfaces well defined differential operators can be used to compute standard functions as e.g. curvatures, tangent planes, normals, shape operators. These differentials cannot be applied directly to digitized surfaces or digitized curves. Consider as a simple example how to compute the tangent in the point $x_{0}$ for the digitized curve $f$ shown in Figure 1. For a continuous curve we would simply calculate the first derivative $d y / d x$. But for the digitized function, the solution is less obvious.

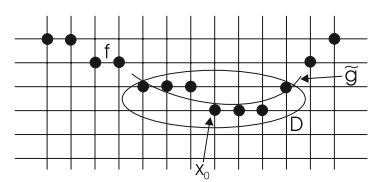

Fig. 1. Finding a tangent to a digitized curve

One could use a continuous fitting function $\tilde{g}$ to approximate the digitized function $f$ in some neighborhood $D$ of $x_{0}$, and then apply the differential operator to that continuous curve. We propose to digitize the differential operator, i.e. to replace it by a 
difference operator that can be applied without problem to digitized as well as continuous functions. This approach also involves the choice of a class of fitting functions, since the selection of the difference operator involves the implicit assumption that there are some continuous functions for which the difference operator yields the correct result. Haralick [1], Langridge [2], Fleck [3], Karabassis and Spetsakis [4] all discuss possible methods to find the best possible fitting functions and difference operators to compute derivatives for a given application.

Digitizing differential operators requires two important choices. The first choice considers which class of fitting functions to use. This choice is not unique, e.g. if we choose linear fitting functions, then all second and higher order differences will vanish. Second, we must choose an appropriate difference operator for each particular class of fitting functions and also this choice is not unique. If we choose a linear fitting function $\tilde{g}(x)=a x+b$, and if we let $\sigma$ denote the shift operator defined by $\sigma^{j} \tilde{g}(x)=\tilde{g}(x+j)$, then we can replace the differential $d / d x$ by one of the three difference operators $\Delta=\left(\sigma^{1}-1\right),\left(\sigma^{2}-1\right) / 2$, or $\left(\sigma^{3}-1\right) / 3$. These operators will all yield the same result when applied to $\tilde{g}(x): d \tilde{g} / d x=\triangle \tilde{g}=a$. Hence there is no straightforward choice for the fitting functions and the difference operator.

In this paper, we show how to choose the appropriate difference operator for each class of fitting functions. We actually avoid the problem of computing the fitting functions by verifying whether the digitized function has the right features to be categorized in one of the function classes. Moreover, feature detection arises in a natural way if we want to compute differentials for digitized functions. The computation of the difference operator and the feature detectors for the function classes both fit in a rigid mathematical framework [5].

We propose a method to choose the most appropriate difference operator with a decision tree, considering the local features of the image. The framework of the method is illustrated by the computation of a digitized difference operator for the Laplacian. Lachaud et al [6] discuss how to estimate the tangent of a digital curve. Lindeberg [7] discusses how to define discrete derivative approximations for the computations of multi-scale low-level feature extraction, and their use in edge detection. Gunn [8] and Demigny et al [9] consider discrete versions of edge detection algorithms. Lowe [10] uses an approximation for the Laplacian of Gaussian to detect stable keypoints. In this paper we show how the Laplacian of Gaussian edge detector can benefit from locally applying the appropriate version of the Laplacian difference operator.

In section 2, we show how to digitize the differential operator for different classes of fitting functions. Next, a decision tree for the practical computation of difference operators is introduced in Section 3, where the computation of the feature detection templates and the difference operators for the Laplacian is given as an example. Section 4 presents the Laplacian of Gaussian edge detector as a practical application to illustrate the improvements by our method. Finally, we conclude this paper in Section 5 .

\section{Digitizing Differential Operators}

When choosing a class of fitting functions for a digitized function, we choose in fact a class of features. We use features to examine how the digitized function behaves locally, 
that is to verify how smooth the function is. Next, we choose one particular difference operator, depending on the detected features. In the remainder of this section we show how to make the appropriate choice of fitting function and its corresponding difference operator. We start by introducing the notations and the conventions used in this paper.

Fitting Functions and Difference Operators. We will use a continuous real function $\tilde{g}: \mathbb{R}^{m} \rightarrow \mathbb{R}$ to approximate a digitized function $f: \mathbb{Z}^{m} \rightarrow \mathbb{Z}$. To approximate the value of a differential at a point $x_{0}$, it is sufficient to approximate $f$ in a finite subset $D \subset \mathbb{Z}^{m}$ containing $x_{0}$. We write $|f-\tilde{g}|<\epsilon$ as a shorthand for $|f(x)-\tilde{g}(x)|<\epsilon$ for all $x \in D$.

The shift operator $\sigma^{j}$ is defined by $\sigma^{j} f(x)=f(x+j)$, for $x, j \in \mathbb{Z}^{m}$. The functional composition of shift operators can be expressed as a multiplication of polynomials, i.e. $\sigma^{j} \sigma^{k} f=\sigma^{j+k} f$. A difference operator $P$ can be represented as a polynomial in $\sigma$, that is $P=\sum_{j=0}^{l} p_{j} \sigma^{j}$. If we write that $P \tilde{g}=0, \sum p_{j} \sigma^{j} \tilde{g}(x)=0$ for all $x, j \in \mathbb{Z}^{m}$. If we write that $|P f-P \tilde{g}|<\epsilon$, this means that $|P f(x)-P \tilde{g}(x)|<\epsilon$ for all $x$ for which $P f(x)$ is well defined, that is $(x+j) \in D$ for every non-vanishing coefficient $p_{j}$ of the difference operator $P$.

We will use the ideal $I$ generated by a set of difference operators $P_{i}$. We write $I=<$ $P_{1}, P_{2}, \ldots>$, and we say that the polynomials $P_{i}$ form a basis for the ideal $I$. $I$ consists of all operators $P=\sum_{i} S_{i} P_{i}$, where the $S_{i}$ are arbitrary polynomials in $\sigma$.

Difference operators can also be represented by their templates. A two-dimensional difference operator $P=\sum p_{j} \sigma^{j}=\sum_{j_{x}, j_{y}} p_{j_{x} j_{y}} \sigma_{x}^{j_{x}} \sigma_{y}^{j_{y}}, j \in Z^{2}$ is represented by a two-dimensional template:

$$
\begin{array}{|l|l|l|}
\hline p_{00} & p_{10} & p_{20} \\
\hline p_{01} & p_{11} & p_{21} \\
\hline p_{02} & \ldots & \\
\end{array}
$$

We use the convention that the box at the upper left corner corresponds to $p_{00}$. Boxes with vanishing coefficients are either not drawn, or drawn as empty boxes.

Approximation of Differentials. Let $L$ be the differential operator that we want to approximate by a difference operator, which can be applied to some digitized function $f$. We find such an approximation by selecting a class $G$ of fitting functions $\tilde{g}$, and a difference operator $Q$ that works well for this class. We must choose in some way a class $G$ of continuous functions and a difference operator $Q=\sum q_{j} \sigma^{j}$, such that $Q \tilde{g}=L \tilde{g}$ for every $\tilde{g} \in G$.

If $\tilde{g}$ is an approximation for $f$ such that $|f-\tilde{g}|<\epsilon$, then we have $|Q f-L \tilde{g}|<$ $\epsilon \sum\left|q_{j}\right|[5]$. Hence, the difference operator $Q$ will be a good approximation for the differential operator $L$ provided $G$ contains at least one function $\tilde{g}$ that is a good approximation for $f$.

Additional Constraints on Fitting Functions. It remains uncertain whether the class $G$ of fitting functions contains a good approximation $\tilde{g}$ for the digitized function $f$. The uncertainty is removed by imposing additional constraints on $G$. We demand that fitting functions $\tilde{g}$ satisfy a (possibly infinite) set of difference equations $P_{i} \tilde{g}=0$, for 
$i=1,2, \ldots$. The operators $P_{i}$ will be used to eliminate the fitting function $\tilde{g}$ from the inequality $|f-\tilde{g}|<\epsilon$. However, the set of operators that can be used to eliminate $\tilde{g}$ is much larger. For any operator $P$ in the ideal $I=<P_{1}, P_{2}, \ldots>, P \tilde{g}=0$. So also $P_{1} P_{2} \tilde{g}=0,\left(P_{1}+P_{2}\right) \tilde{g}=0$, and $\left(\sum S_{i} P_{i}\right) \tilde{g}=0$ for arbitrary difference operators $S_{i} \in \mathbb{R}[\sigma]$.

We will assume from now on that we have chosen a class of fitting functions $G$, by specifying a set of difference operators $P_{i}$, i.e. $G=\left\{\tilde{g} \mid P_{i} \tilde{g}=0\right.$, for $\left.i=1,2, \ldots\right\}$. We can then prove that there is a fitting function $\tilde{g} \in G$ such that $|f-\tilde{g}|<\epsilon$ if and only if

$$
|P f|<\epsilon \sum\left|p_{j}\right|
$$

for every operator $P$ in the ideal $I$ generated by all $P_{i}$, i.e. $I=<P_{1}, P_{2}, \ldots>[5]$.

The ideal $I$ of difference operators is generated by a possibly infinite set of operators $P_{i}$. Hilbert's Basis Theorem for polynomial ideals states that any ideal of polynomials in the ring $\mathbb{R}\left[\sigma_{x}, \sigma_{y}\right]$ can always be generated by a finite basis of polynomials. Even if a system has infinitely many difference equations, these can all be obtained by multiplying, adding and translating a finite set of basis equations. We can compute a Groebner basis for any ideal if we impose an ordering on the shift operators $\sigma_{x}, \sigma_{y}$ [12]. For each different class of fitting functions, a Groebner basis completely characterizes all different templates that will recognize a function of that class. The Groebner basis is used in efficient algorithms for determining whether a given polynomial belongs to an ideal. The functional decomposition and combination of feature detectors can also be investigated using these bases [5].

How to Compute Feature Detectors for a Function Class? The introduction of the difference equations $P_{i} \tilde{g}=0$ has an important consequence: by eliminating the explicit occurrence of $\tilde{g}$, the use of fitting functions will be replaced by the use of feature detectors $P_{i}$. Instead of actually fitting a good continuous approximation $\tilde{g}$ to $f$, we must verify whether Eq.2 2 holds for every difference operator in $I$, i.e. whether $f$ has the right features. So we do not bother which fitting function would actually yield the closest fit.

Features can be detected without error by verifying only a finite number of inequalities, when the solution space of the difference equations is a finite linear vector space. Assume that the solution set of the partial difference equations $P_{1} g=0, \ldots, P_{n} g=0$ can be written as a linear vector space with $g_{1}, \ldots, g_{l}$ as a basis:

$$
\alpha_{1} g_{1}+\cdots+\alpha_{l} g_{l}
$$

Let $K_{D}$ be the set of all difference operators $P_{i}$ of the form

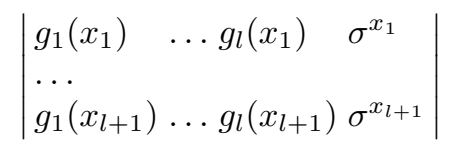

with the points $x_{j} \in D$. The operators of $K_{D}$ are written as determinantal expressions of the coefficients $g_{j}\left(x_{j}\right)$ and the shift operators $\sigma^{x_{j}}$. Let $I_{D}$ denote the set of all the difference operators in $I$ for which $P_{i} f(x)$ is well defined for at least one $x$ in $D$, i.e. $(x+j) \in D$ for every non-vanishing coefficient $p_{j}$ of the difference operator $P_{i}$. 
Thus the neighborhood $D$ must be large enough such that $I_{D}$ is equal to $I$, i.e. $I_{D}$ must contain a basis for $I$. Then the polynomials of $K_{D}$ form a finite basis for the ideal generated by $I_{D}$. Furthermore, if the function $f$ satisfies the inequality $\left|P_{i} f\right|<\epsilon \sum\left|p_{j}\right|$ for every polynomial $P_{i}$ in $K_{D}$, then $f$ will satisfy this inequality for all polynomials of $I_{D}$ [11]. In general, a small sample of $K_{D}$ suffices to calculate a Groebner basis for the ideal $I$.

How to Choose a Difference Operator for a Function Class? For any difference operator $Q$ chosen such that $Q \tilde{g}=L \tilde{g}$, and $f$ satisfying $|P f|<\epsilon \sum\left|p_{j}\right|$ for all $P \in I=<$ $P_{1}, \ldots, P_{n}>$, there exists a function $\tilde{g}$ satisfying $P \tilde{g}=0$ such that

$$
|Q f-L \tilde{g}|<\epsilon \sum\left|q_{j}\right| \text {. }
$$

This result states exactly what we propose: the combined use of feature detectors $P$ and a difference operator $Q$, linked to each other by the fitting functions $\tilde{g}$. It is sufficient to verify whether $f$ has the right features so that we can apply this particular difference operator $Q$. However, as mentioned before, the choice of $Q$ is not unique.

How to Choose the Best Difference Operator $Q$ ? Once the class of fitting functions has been chosen by verifying Eq. 2, we must choose a difference operator $Q$, which satisfies $Q \tilde{g}=L \tilde{g}$. Since $(Q+P) \tilde{g}=Q \tilde{g}$, there seem to be many possible ways to choose $Q$. There are however no other possibilities than those provided by the ideal $I$. Every operator $R$ satisfying $R \tilde{g}=Q \tilde{g}$ can be written as $R=Q+P$.

Among all operators $R$, we can in fact look for the best candidate. For any operator $P \in I$ we have

$$
|(Q+P) f-L \tilde{g}|<\epsilon \sum\left|p_{j}+q_{j}\right|
$$

The difference operator $P$ for which the right side of the above inequality becomes minimal, gives the lowest error in $P+Q$ when used to approximate the differential $L$.

As $P_{i} \tilde{g}=0$ for every $P_{i} \in I$, we can look for an operator of the form

$$
O=Q+\sum\left(S_{i} P_{i}\right)
$$

where $S\left(\sigma_{x}\right)$ is an arbitrary difference operator. Then the following inequality

$$
|O f-L \tilde{g}|<\epsilon \sum\left|o_{j}\right|
$$

must be satisfied and the best difference operator is the one for which $\epsilon \sum\left|o_{j}\right|$ is minimized. This gives a systematic method for computing the difference operator to use for a particular fitting function class.

\section{A Decision Tree for the Laplacian Operator}

In this section we apply the above theory in the design of difference operators for the computation of the Laplacian $\partial^{2} / \partial x^{2}+\partial^{2} / \partial y^{2}$. We want to adapt the widely used classical Laplacian kernel to yield better results once the local image characteristics 
are known. To determine the correct fitting function class in each point on the image surface, we use a decision tree as shown in Figure 2. Each node of the decision tree shows a Groebner basis for the feature detectors for a particular function class. Once the correct fitting function is chosen, the appropriate difference operator will be selected in the underlying leaf of the tree.

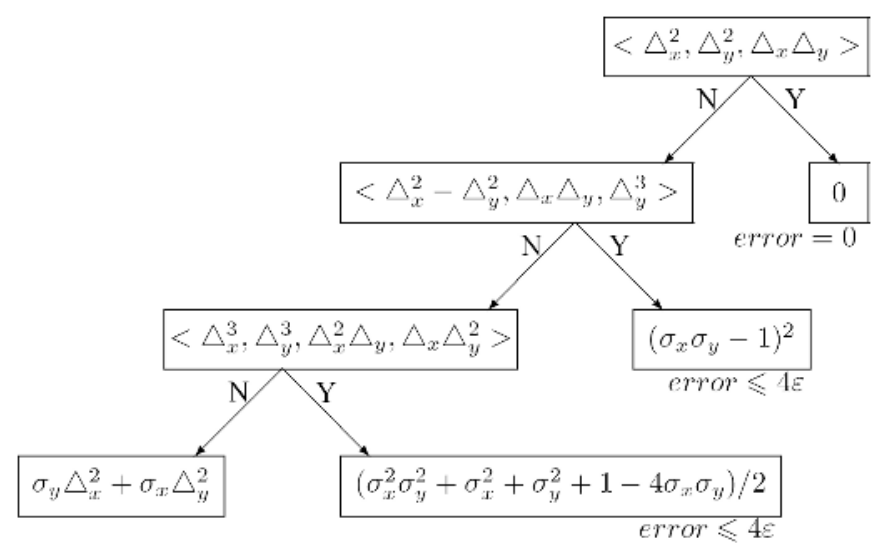

Fig. 2. A decision tree for the Laplacian

\subsection{Template Bases for Feature Detectors}

The first goal is to determine which fitting function is locally the best approximate for the digitized image surface. The decision tree uses three fitting function classes:

$$
\begin{aligned}
& \alpha_{1} x+\alpha_{2} y+\alpha_{3} \\
& \alpha_{1}(x+y)^{2}+\alpha_{2} x+\alpha_{3} y+\alpha_{4} \\
& \alpha_{1} x^{2}+\alpha_{2} y^{2}+\alpha_{3} x y+\alpha_{4} x+\alpha_{5} y+\alpha_{6}
\end{aligned}
$$

A set of feature detection templates is computed for each fitting functions as the polynomials of $K_{D}$ in Eq.4 This is illustrated for the function class used in the second node of the decision tree: $\alpha_{1}\left(x^{2}+y^{2}\right)+\alpha_{2} x+\alpha_{3} y+\alpha_{4}$. In this case, the polynomials of the set $K_{D}$ have the form

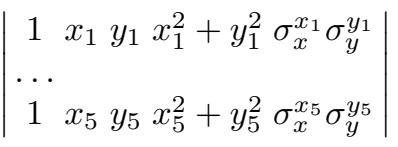

For example, for $\left\{\left(x_{1}, y_{1}\right), \ldots,\left(x_{5}, y_{5}\right)\right\}=\{(0,0),(1,1),(2,0),(3,1),(4,0)\}$, the above determinant is equal to $1-2 \sigma_{x} \sigma_{y}+2 \sigma_{x}^{3} \sigma_{y}-\sigma_{x}^{4}$ which corresponds to the template

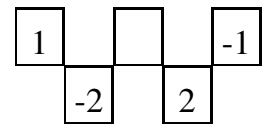


Thus a basis for the ideal $I_{D}$ for each function class can be generated systematically by computing all polynomials of $K_{D}$. Based on a limited set of polynomials we can compute $<\left(\sigma_{x}-1\right)^{2}-\left(\sigma_{y}-1\right)^{2},\left(\sigma_{x}-1\right)\left(\sigma_{y}-1\right),\left(\sigma_{y}-1\right)^{3}>$, i.e. $<\triangle_{x}^{2}-\triangle_{y}^{2}, \triangle_{x} \triangle_{y}, \triangle_{y}^{3}>$ as a Groebner basis for this function class. The corresponding templates are

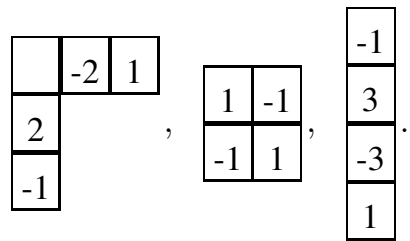

For each of the fitting function classes, the Groebner basis completely characterizes the templates that will recognize the function. Table 1 lists a selection of functions and their corresponding Groebner bases. Figure 3 shows the decision tree of Figure 2 with templates for the Groebner basis for the ideal of each function class. The nodes of the decision tree are practically implemented as a finite set of inequalities to be verified for the function of the required form. Or, equivalently, templates are generated that can detect features for each of the fitting functions. Now that we have an implementation for the nodes, the leaves of the tree remain to be filled in with the appropriate difference operators.

Table 1. Functions and corresponding Groebner bases with lexicographic ordering $\Delta_{x}>\Delta_{y}$

\begin{tabular}{|l|l|}
\hline$\alpha_{1}$ & $<\Delta_{x}, \Delta_{y}>$ \\
$\alpha_{1} x+\alpha_{2}$ & $<\Delta_{x}^{2}, \Delta_{y}>$ \\
$\alpha_{1}(x+y)+\alpha_{2}$ & $<\Delta_{x}-\Delta_{y}, \Delta_{y}^{2}>$ \\
$\alpha_{1} x+\alpha_{2} y+\alpha_{3}$ & $<\Delta_{x}^{2}, \Delta_{x} \Delta_{y}, \Delta_{y}^{2}>$ \\
$\alpha_{1} x y+\alpha_{2} x+\alpha_{3} y+\alpha_{4}$ & $<\Delta_{x}^{2}, \Delta_{y}^{2}>$ \\
$\alpha_{1}(x+y)^{2}+\alpha_{2}(x+y)+\alpha_{3}$ & $<\Delta_{x}-\Delta_{y}, \Delta_{y}^{3}>$ \\
$\alpha_{1}\left(x^{2}+y^{2}\right)+\alpha_{2} x+\alpha_{3} y+\alpha_{4}$ & $<\Delta_{x}^{2}-\Delta_{y}^{2}, \Delta_{x} \Delta_{y}, \Delta_{y}^{3}>$ \\
$\alpha_{1}(x+y)^{2}+\alpha_{2} x+\alpha_{3} y+\alpha_{4}$ & $<\Delta_{x}^{2}-\Delta_{y}^{2}, \Delta_{y}\left(\Delta_{x}-\Delta_{y}\right), \Delta_{y}^{3}>$ \\
$\alpha_{1} x^{2}+\alpha_{2} y^{2}+\alpha_{3} x+\alpha_{4} y+\alpha_{5}$ & $<\Delta_{x}^{3}, \Delta_{x} \Delta_{y}, \Delta_{y}^{3}>$ \\
$\alpha_{1} x^{2}+\alpha_{2} y^{2}+\alpha_{3} x y+\alpha_{4} x+\alpha_{5} y+\alpha_{6}$ & $<\Delta_{x}^{3}, \Delta_{x}^{2} \Delta_{y}, \Delta_{x} \Delta_{y}^{2}, \Delta_{y}^{3}>$ \\
\hline
\end{tabular}

\subsection{Difference Operators}

Now that the template bases for the polynomial ideals are known, the next step is to determine the best difference operator for each class of fitting functions. As an example we describe the computation of the difference operator for quadratic functions of the form $\alpha_{1} x^{2}+\alpha_{2} y^{2}+\alpha_{3} x y+\alpha_{4} x+\alpha_{5} y+\alpha_{6}$. We must choose $Q$ so that the requirement $L \tilde{g}=Q \tilde{g}$ is satisfied, that is

$$
\left(\frac{\partial^{2}}{\partial x^{2}}+\frac{\partial^{2}}{\partial y^{2}}\right) \tilde{g}=\left(\triangle_{x}^{2}+\triangle_{y}^{2}\right) \tilde{g}=2 \alpha_{1}+2 \alpha_{2} .
$$

For this function class, any differential operator of the form

$$
O\left(\sigma_{x}, \sigma_{y}\right)=\triangle_{x}^{2}+\triangle_{y}^{2}+\triangle_{x}^{3} S_{1}+\triangle_{y}^{3} S_{2}+\triangle_{x}^{2} \triangle_{y} S_{3}+\triangle_{x} \triangle_{y}^{2} S_{4}
$$


in which $S_{i}$ are arbitrary polynomials in the shift operators, yields the exact value for the Laplacian. For optimal results, one must choose the operator of the form (14) for which $\sum\left|o_{j}\right|$ is as small as possible. For this function class, the best choice is the symmetric operator

$$
\frac{\sigma_{x}^{2} \sigma_{y}^{2}+\sigma_{x}^{2}+\sigma_{y}^{2}+1-4 \sigma_{x} \sigma_{y}}{2} .
$$

The difference operators for the other function classes are obtained in a similar way, and their templates are shown in Figure 3.

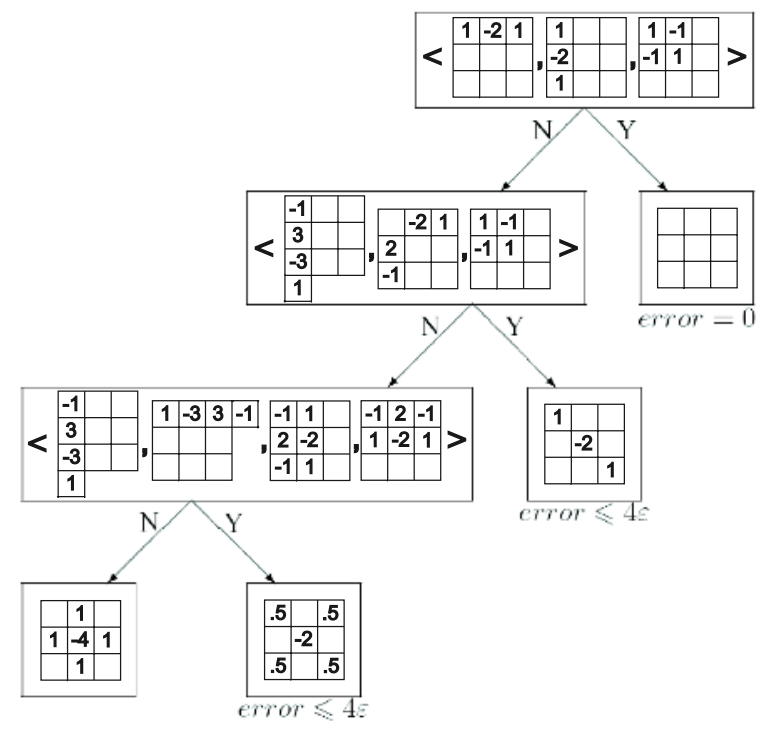

Fig. 3. A decision tree for the Laplacian

The obtained difference operators lead to some interesting conclusions. Without feature detection, we would always choose the classical discrete equivalent of the Laplacian as shown in the lower left leaf of the decision tree. When the digitized function is locally linear or close to linear, the best possible difference operator is the zero operator, yielding an error equal to zero. In fact, it is known without uncertainty that the Laplacian of a linear function vanishes. Since difference operators for linear functions do not have to compensate for quadratic terms, they perform better than the difference operators derived for quadratic functions. For both quadratic functions, we found a difference operator which yields a maximal error of $4 \epsilon$ on the computed value of the Laplacian. For quadratic functions with circular symmetry (the second level in the decision tree), it is sufficient to compute the second order difference in a diagonal direction. Finally, for quadratic functions the best difference operator has a template equal to the classical discrete Laplacian operator rotated over 45 degrees and divided by 2 . If none of the feature detection tests succeed, we use the classical discrete Laplacian operator. In fact, the differences between the operators appear to be quite small, but the examples in section 4 show that considerable improvements are obtained by using the decision tree. 


\section{Laplacian of Gaussian Edge Detection}

We illustrate the practical use of the decision tree for the computation of the Laplacian of Gaussian (LoG) for edge detection. The LoG is computed by first convolving the image with a Gaussian of a certain width and then passing a Laplacian filter kernel over the Gaussian smoothed image. The edges are computed as the zero-crossings of the Laplacian. We compare the results for the computation of the Laplacian by our method to the computation with the classical version of the kernel. Our method uses the decision tree designed above to select the most appropriate Laplacian operator in each image point. First, the local characteristics of the digitized image surface are determined by subsequently verifying a set of inequalities for each class of fitting functions. Once the correct fitting function is determined, the corresponding difference operator can be applied. For each image in the experiment, we constructed a scale space for different widths, i.e. different values for the standard deviation, of the Gaussian, so that we can compare both methods on increasing levels of smoothing. The results for the edge detection by both methods are shown in Figure4

Are the Fitting Function Classes Useful? For each function class, a set of inequalities is created to detect the local features of the digitized image. When the image surface

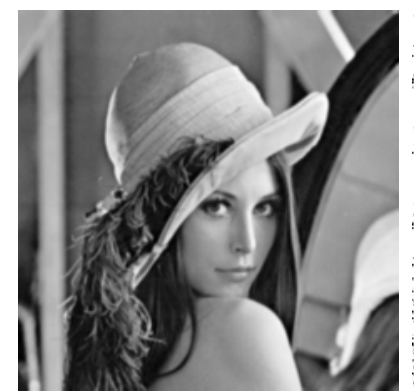

$(1 a)$

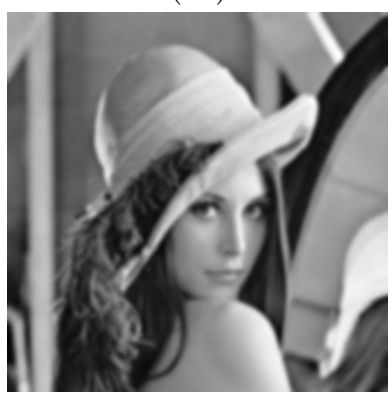

$(2 a)$

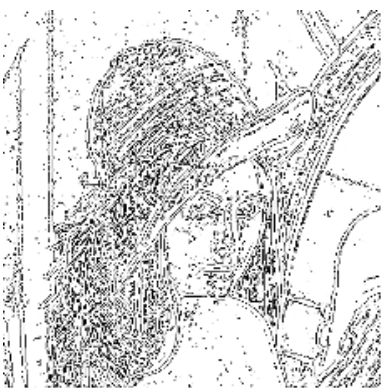

(1b)

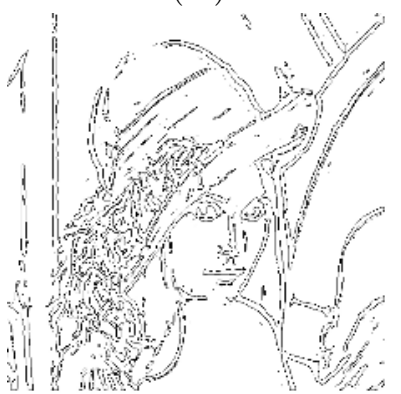

$(2 b)$

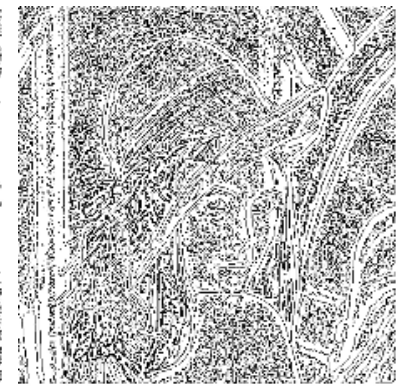

$(1 c)$

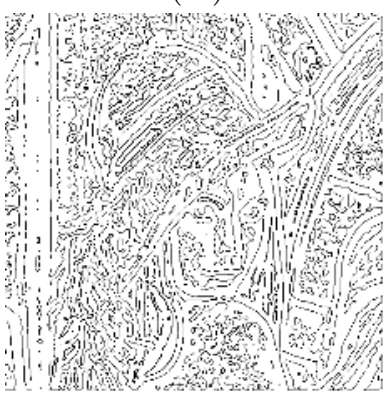

$(2 c)$

Fig. 4. LoG edge detection: In column $a$ we see the Gaussian smoothed images on different scales, respectively for standard deviation $\sqrt{2}$ and $2 \sqrt{2}$. Column $b$ shows the result of edge detection after application of the decision tree to compute the Laplacian. Column $c$ shows the result of edge detection after application of the classical Laplacian kernel. 


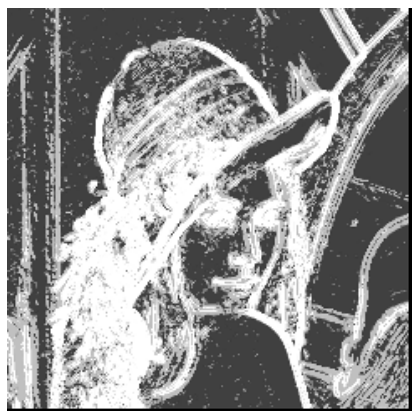

Fig. 5. The fitting function class in each image point is indicated: the higher (whiter) the point's intensity, the lower the node of the fitting function in the decision tree of Figure 3 When no correct approximation is found, points are colored white. The image was convolved first with a Gaussian of standard deviation $\sqrt{2}$.

locally fulfills the conditions posed by a limited subset of inequalities of the form Eq.2 with $\epsilon=1 / 2$ to account for digitization errors, we consider the fitting function a good approximation for the surface. Figure 5 shows that the three classes are well chosen as nodes for the decision tree. For each pixel, the function class is indicated by one of three gray values: the higher the intensity, the lower the class occurs in the decision tree. It is clear that the image surface is approximated by the expected function class in each image point. When none of the continuous fitting functions approximated the image surface well, e.g. at sharp or discontinuous edges, the classical version of the Laplacian kernel is applied. We cannot define or predict the error on the computation of the Laplacian in such points. These points are indicated by white pixels in Figure 5 Since all gray values appear in Figure 5, all nodes of the decision tree are useful.

What Is the Size of the Set of Feature Detection Templates to be Verified in Each Node of the Decision Tree? The local image features are detected in a neighborhood $D$ by verifying a set of inequalities of the form Eq. 2 For each node of the decision tree, we generate a set $K_{D}$ of operators $P$ as in Eq. 4 The number of inequalities, i.e. the size of the set $K_{D}$, is then given by the binomial $\left(\begin{array}{l}n \\ l\end{array}\right)$, with $n$ the number of points in the neighborhood $D$ and $l$ the number of basis vectors in the solution set of $P_{i} \tilde{g}=0$ (Eq. 3). Verifying a set of that size at each node yields a rather large computational complexity for the decision tree. The complexity can be reduced by verifying subsets with a lower number of inequalities at each node. Simulations show that the classification in function classes does not significantly vary with the subset's size. The edge detection results do not noticeably differ, and satisfying results are obtained for rather small subsets of inequalities. The results shown in Figure 4 are obtained with a subset of seven feature templates per node. However, selecting only a limited subset cannot be done without introducing a small error. More on this subject can be found in [5, 13].

The computational complexity of the decision tree is further reduced by using feature templates in more than one neighborhood $D$. The computation of the templates is dependent on the size of $D$, but most of the polynomial's coefficients are zero, as can be seen in Eq.11. So the majority of the results for the verification of inequalities can 
be reused in adjoining neighborhoods. To avoid systematic errors, introduced by using the same subset of templates over and over for all points, we randomly choose a new subset for every neighborhood to complement the reused results.

Comparison of Methods. The Laplacian computed with the proposed decision tree is compared to the result of applying the classical Laplacian kernel. The experiments are repeated in a scale space of Gaussians with increasing standard deviation. Figure 4 shows the results for Gaussians with standard deviation $\sqrt{2}$ and $2 \sqrt{2}$. The results for the LoG edge detection are considerably better when the Laplacian is computed with the decision tree. The most significant edges are detected and the edges of important details are preserved, as the images in column $(b)$ show. If we compare this to the result in column $(c)$, we notice an abundance of edges in image regions considered homogeneous. Even on a higher scale, i.e. for even smoother images, the computation of the Laplacian with the classical kernel does not yield better results. First, the error on the localization of edges increases for higher levels of smoothing. Second, the edges of finer (and even coarser) details disappear on higher scales while edges are still detected in (noisy) homogeneous regions. Both problems are avoided in our method. Edges of details are already distinguished on finer scales. If linear fitting functions can locally approximate the image surface in homogeneous regions, the zero operator is used for the Laplacian so that zero crossings do not occur in these regions. Note that some drawbacks of LoG edge detection are apparent in the results of both methods, edges tend to form closed loops, and sharp corners are smoothed too much.

\section{Conclusion}

We present a mathematical framework from which both feature detection and difference operators arise in a natural way. By detecting local image features, we avoid the necessity of actually approximating the digitized image surface by fitting functions. For each function class, we define the appropriate difference operator which yields a minimal computational error when approximating the value we would have obtained by the differential operator.

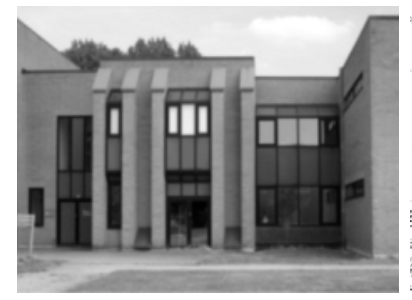

(a)

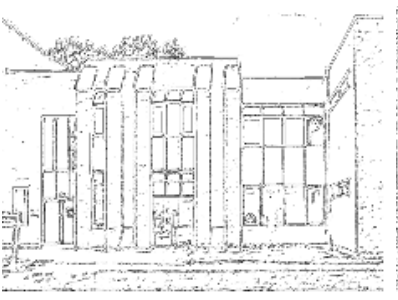

(b)

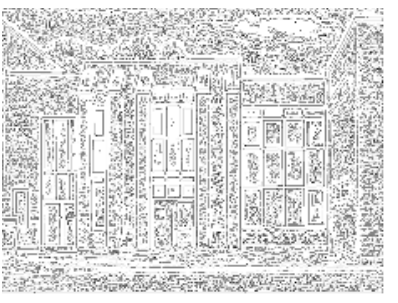

(c)

Fig. 6. LoG edge detection: Image $(a)$ shows an Gaussian smoothed image with standard deviation $2 \sqrt{2}$. Image $(b)$ shows the result of edge detection after application of the decision tree to compute the Laplacian. Image $(c)$ shows the result of edge detection after application of the classical Laplacian kernel 
We conclude that the quality of the edge pixels detected by the LoG improves when the Laplacian difference operator is adapted to the knowledge about the local image features. Clearly, a practical application like the computation of straight lines and corners of a building as in Figure $6(a)$, is considerably easier when given the LoG edge pixels of Figure 6 $(b)$ (decision tree) as opposed to the information obtained by the default kernel, shown in Figure 6 $(c)$.

\section{References}

1. Haralick, R.: Digital step edges from zero crossing of second directional derivatives. IEEE Trans. Pattern Anal. Machine Intell. 6 (1984) 58-68

2. Langridge, D.: Detection of discontinuities in the first derivatives of surfaces. Comput. Vision Graphics Image Process. 27 (1984) 291-308

3. Fleck, M.: Multiple widths yield reliable finite differences. IEEE Trans. Pattern Anal. Machine Intell. 14 (1992) 412-429

4. Karabassis, E. and Spetsakis, M.E.: An analysis of image interpolation, differentiation, and reduction using local polynomial fits. CVGIP: Graphical Models and Image Processing 57 (1995) 183-196

5. Veelaert, P.: Local feature detection for digital surfaces. In: Proceedings of the SPIE Conference on Vision geometry V, SPIE Vol. 2826 (1996) 34-45

6. Lachaud, J.O., Vialard, A. and de Vieilleville, F.: Analysis and Comparative Evaluation of Discrete Tangent Estimators. In: Proc. DGCI'05, LNCS, Vol. 3429 (2005) 240-251

7. Lindeberg, T.: Discrete Derivative Approximations with Scale-Space Properties: A Basis for Low-Level Feature Extraction. J. of Mathematical Imaging and Vision 3 (1993) 349-376

8. Gunn, S.: On the discrete representation of the Laplacian of Gaussian. Pattern Recognition 32 (1999) 1463-1472

9. Demigny, D. and Kamlé, T.: A Discrete Expression of Canny's Criteria for Step Edge Detector Performances Evaluation. IEEE Trans. Patt. Anal. Mach. Intell. 19 (1997) 1199-1211

10. Lowe, D.G.: Distinctive image features from scale-invariant keypoints. International Journal of Computer Vision 60(2) (2004) 91-110

11. Stoer, J. and Witzgall C.: Convexity and Optimization in Finite Dimensions I. Springer, Berlin (1970)

12. Cox, D., Little, J. and O'Shea, D.: Ideals, Varieties and Algorithms: an Introduction to Computational Algebraic Geometry and Commutative Algebra. Springer, New York (1992)

13. Veelaert, P. and Teelen K.: Fast polynomial segmentation of digitized curves, Accepted for DGCI 2006. 